\title{
Radial Photic Stimulation for Maximal EEG Response for BCI Applications
}

\author{
Surej Mouli \\ School of Computing \\ University of Kent \\ Email: surej@ieee.org
}

\author{
Ramaswamy Palaniappan \\ School of Computing \\ University of Kent \\ Email: r.palani@kent.ac.uk
}

\begin{abstract}
This study proposes the use of radial visual stimuli design to obtain increased steady state visual evoked potential (SSVEP) responses that can be utilised in brain-computer interfaces (BCI). Visual stimuli designs based on chip on board (COB) LEDs were used in this study to compare the influences of the radial with horizontal and concentric patterns in SSVEP. Circular rings with diameters $70 \mathrm{~mm}, 90 \mathrm{~mm}, 110 \mathrm{~mm}$, and 130 $\mathrm{mm}$ with green COB LEDs were used for radial and concentric patterns while green strip COB LED of $18 \mathrm{~mm}$ width and length $16 \mathrm{~cm}$ was used for horizontal pattern. The visual flicker and the concentric patterns were generated and controlled precisely by a 32-bit microcontroller platform. The SSVEP frequencies used were $7,8,9$ and $10 \mathrm{~Hz}$ for a period of 30 seconds for each horizontal, radial and concentric circle visual stimulus. The study analysed the EEG recording from five participants comprising of five trials from each frequency and three different stimuli designs to identify the most responsive visual stimulus for evoking SSVEP. Furthermore, we also compared the influence of ring diameters in radial visual stimulus to identify the maximal response and minimal visual fatigue. The results indicated that radial stimulus gave significantly better response than concentric circles or horizontal stimuli for all the participants. Also $130 \mathrm{~mm}$ radial stimulus gave the highest response and viewing comfort.
\end{abstract}

Keywords - Brain-computer Interface; Concentric; Electroencephalogram; LED; Radial; Steady State Visual Evoked Potential; Visual Stimulus.

\section{INTRODUCTION}

B RAIN waves recorded from the scalp using non-invasive methods with electroencephalography (EEG) is a standard tool used in various mental health related diagnosis [1]-[3]. It is also becoming a more popular method in multi-disciplinary studies combining neuroscience, psychology, computer science and engineering for various applications [4], [5]. EEG based brain-computer interface (BCI) is a type of human-machine communication system utilising brain signals to control external applications or devices. BCI research is widely explored in recent decades and such technologies can assist people with disabilities to provide communication capabilities to relay their intentions to external world through a non-muscular medium [5]-[8].

EEG based BCI is popular due its low cost, portability and high temporal resolution. The data is collected using sensors placed at various positions around the head based on the requirement of the study or application. BCI applications are generally developed using EEG signals based on P300 potential or steady state visual evoked potential (SSVEP) as these give higher success rates. However motor imagery based BCI is yet another possible approach [9]-[12].

Figure 1 shows an example of EEG data acquisition, wireless data transfer and data processing in a BCI based application control system. The data can be recorded using wireless Emotiv EPOC+, which wirelessly transmits the data to the computer using proprietary protocol with real time monitoring of packet loss and connection quality. The raw EEG recordings are processed and classified for controlling external applications or other non-muscular communication systems.

In this paper, radial stimulus were studied along with horizontal and concentric designs for SSVEP based BCI application with the aim of increasing the response. SSVEP is a repetitive sinusoidal like signal generated in the brain when the user focuses on a flickering light which flashes continuously at a constant frequency [10], [11], [13]-[15].

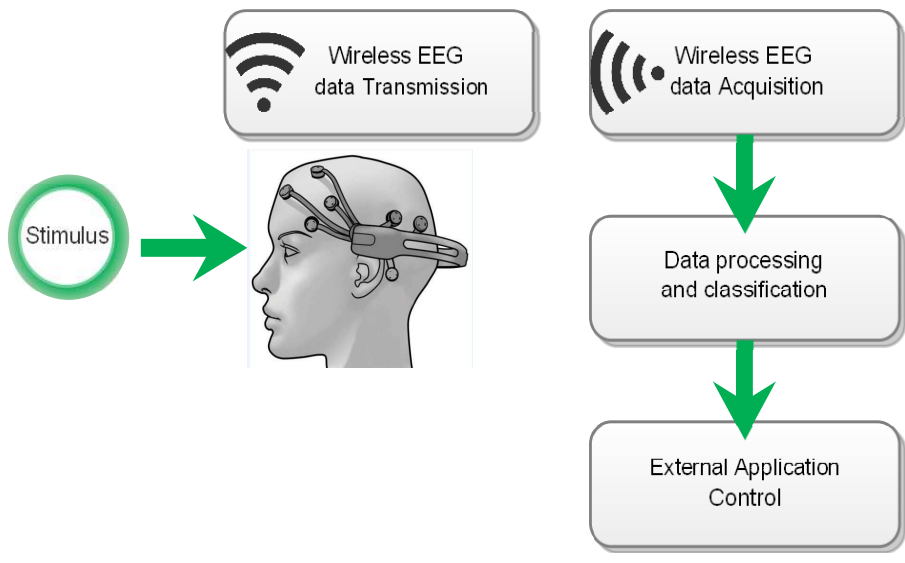

Fig. 1. Basic BCI control system

SSVEP can be evoked using a visual stimulus based on programmed flashes on LCD screen or using LEDs, flashing with precise frequency as per requirement. SSVEP based BCI systems are widely used due to the high information 
transfer rate, little training and the suitability for practical BCI applications. In spite of all these advantages, SSVEP is not always comfortable for prolonged usage due to visual fatigue for the user [16]. Previous studies have explored the effect of colour and orientation of the visual stimulus for improving the responses in SSVEP designs and also to reduce the visual fatigue [9], [14], [16], [17]. The horizontal LED array design has been shown to give improved response over vertical and single LED [9].In this proposed study, we used chip-on-board (COB) LED visual stimulus in SSVEP elicitation as it improves the LED lighting quality and has uniform light distribution [18]. The proposed radial visual stimulus was designed using a single ring of green COB LED's with diameters of $70,90,110$ and $130 \mathrm{~mm}$ as this was the sizes available in the market. Green colour was chosen based on results of previous study [14]. The concentric stimulus was designed using green COB radial LED rings but with progressive motion as explained in Section II. The horizontal stimulus was designed using green COB LED strip of $18 \mathrm{~mm}$ wide and $160 \mathrm{~mm}$ long.

The frequencies used in this study were $7,8,9$ and 10 $\mathrm{Hz}$ as the lower frequencies gave better response [14]. The frequencies were generated by a 32 bit microcontroller platform with LED's driven by MOSFETs for constant brightness. The complete system was powered by a $5 \mathrm{~V}$ direct current (DC) source from a battery pack in order to avoid any line interferences from the mains supply. The EEG responses from all three visual stimuli were compared and analysed to identify the visual stimulus design that has the highest performance and reduced visual fatigue. The experiment also investigated the influence of ring diameters in radial visual stimulus, for evoking SSVEP response and also comfort level for the user.

\section{MATERIAL AND METHODS}

\section{A. Experimental Setup}

To explore the influences of radial, concentric and horizontal stimuli on SSVEP, five participants(It is a pilot study and hence only five participants) with perfect or corrected vision were chosen in the age group of 24 to 45 (two males and three females). The participants were seated $60 \mathrm{~cm}$ from the visual stimulus which was placed at eye level. The participants did not have any prior experience with BCI. Written consents were obtained from all the participants after briefing them on the objectives and requirements of the them. The study received ethical approval from Faculty of Sciences Ethics Committee at University of Kent. EEG was recorded using Emotiv EPOC research edition with 14 electrodes, though only a single active electrode located at $\mathrm{O} 2$ was used for this study. The visual stimulus was programmed with the desired frequency to evoke the SSVEP for a period of 30 seconds for each trial. The EEG headset was prepared with all electrodes fitted with saline soaked felts and positioned on participants head and good contact was verified using Emotiv test bench software. The recording process started with horizontal stimulus at $7 \mathrm{~Hz}$ recorded for a period of 30 seconds followed by $7 \mathrm{~Hz} 70$ - $130 \mathrm{~mm}$ radials and $7 \mathrm{~Hz}$ concentric circles each with the same durations. This was repeated for five trials with a total data collection period of 150 seconds. Recording break of one minute was given between each 30 second recording session to allow any previous stimuli influences to subside. The process was repeated for all the other frequencies.

\section{B. Visual Stimulus Design}

This study used visual stimulus based on COB green LEDs consisting of four circular rings with diameters of $70 \mathrm{~mm}, 90$ $\mathrm{mm}, 110 \mathrm{~mm}$ and $130 \mathrm{~mm}$, and one horizontal LED strip of $18 \mathrm{~mm}$ width and $160 \mathrm{~mm}$ length shown in Figure 2. Each LED ring was individually controlled from the microcontroller and driven by a high current MOSFET. The concentric circle stimulus consisted of four circular LED rings flashing at the desired frequency in a sequence of $\mathrm{ON}$ and $\mathrm{OFF}$ fashion to produce the desired frequency. It starts with the $70 \mathrm{~mm}$ ring in active high state for the desired ON period and changes to active low followed by the $90 \mathrm{~mm}$ ring going active for the same period of time as the $70 \mathrm{~mm}$. This progression continues to the $130 \mathrm{~mm}$ ring then repeats from $70 \mathrm{~mm}$ again. This entire process produces a concentric visual stimulus in an expanding fashion.

The LED ON/OFF timing control was designed using a 32 bit ARM Cortex M4 Teensy development board which operates at $72 \mathrm{MHz}$ and can generate frequencies precisely. The block diagram of the schematic is shown in Figure 3. To maintain the optimum brightness throughout the experiments, high current MOSFETs were used for all individual LED rings and horizontal LED strip. The complete system was powered using a 5V DC battery pack with $5000 \mathrm{~mA}$ current capacity to ensure the steady supply of power to the visual stimulus.

The visual stimulus frequencies programmed for the flickers were 7, 8, 9 and $10 \mathrm{~Hz}$ with an accuracy of $0.05 \mathrm{~Hz}$. The precision of the flicker frequencies were confirmed with a digital oscilloscope at the LED terminals.

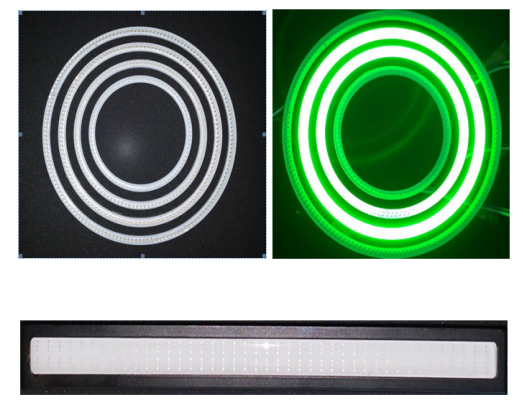

Fig. 2. LED stimuli for radial, concentric circles and horizontal 


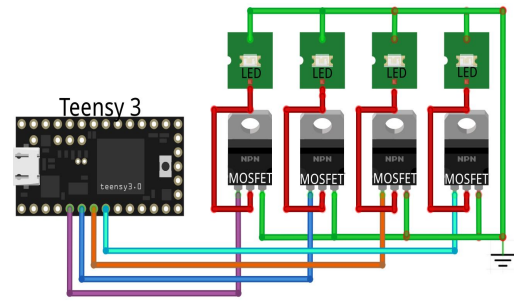

Fig. 3. Teensy 3.2 microcontroller and MOSFET driver

\section{EEG Data Acquisition and Processing}

The EEG data was recorded using Emotiv EPOC research edition with 14 data channels and with two reference electrodes and the layout is shown in Fig 4. In this study, only the data from electrode $\mathrm{O} 2$ was used to analyse the responses of all three visual stimulus. It is generally known that SSVEP is maximal in occipital region and hence the chosen electrode location [19]. The EEG data was recorded using Emotiv test bench software for 30 seconds at $128 \mathrm{~Hz}$ sampling frequency and the data was stored in the European Data Format (EDF). Each participant had five trials for each frequency and stimulus type. The total number of trials for each participant is as shown in Table 1. The total recording time for all the visual stimuli for each participant is 60 minutes (120 trials each lasting 30 seconds). The actual experimental time was slightly longer giving time for breaks and setup. The EDF format EEG data from channel $\mathrm{O} 2$ was converted to MATLAB format for offline analysis using EEGLAB [20].

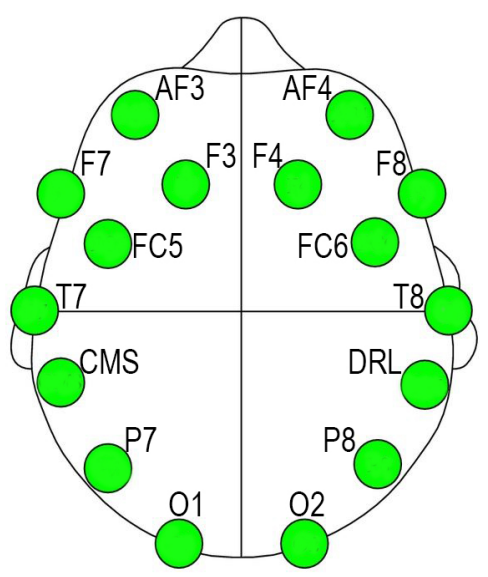

Fig. 4. Emotiv electrode locations [21]

The 30 second recording was filtered with band-pass filter of $2 \mathrm{~Hz}$ bandwidth with centre frequency as the stimulus frequency and data segmented into one second EEG segments. The five trials for each stimulus had 150 segments of one second data which was analysed using Fast Fourier Transform (FFT) and the maximum FFT amplitudes from each segment were stored for statistical analysis.
TABLE I

TRIAL COUNT FOR EACH PARTICIPANT

\begin{tabular}{|c|c|c|c|c|c|c|c|}
\hline $\begin{array}{c}\text { Stimulus } \\
\text { Type }\end{array}$ & $\begin{array}{c}70 \\
\mathrm{~mm}\end{array}$ & $\begin{array}{c}90 \\
\mathrm{~mm}\end{array}$ & $\begin{array}{c}110 \\
\mathrm{~mm}\end{array}$ & $\begin{array}{c}130 \\
\mathrm{~mm}\end{array}$ & Conc & Hori & $\begin{array}{c}\text { Total } \\
\text { Trials }\end{array}$ \\
\hline $\begin{array}{c}\text { Participant } \\
\text { Trials(5 Trials } \\
\begin{array}{c}\text { x } 4 \\
\text { frequencies) }\end{array}\end{array}$ & 20 & 20 & 20 & 20 & 20 & 20 & 120 \\
\hline
\end{tabular}

Statistical analysis was performed using Kruskal-Wallis tests as normality was not assumed. The analysis was performed on maximal FFT amplitudes of EEG data from all radial sizes, concentric circles and horizontal orientation for all four frequencies from five participants.

\section{RESULTS AND DISCUSSIONS}

As mentioned, three different visual stimuli performances were tested for SSVEP responses; (a) radial COB LED stimulus with sizes from 70, 90, 110 and $130 \mathrm{~mm}$, (b) concentric circular patterns in expanding fashion, and (c) horizontal $\mathrm{COB}$ LED stimulus. All the mentioned visual stimuli were analysed for four frequencies 7, 8, 9, and $10 \mathrm{~Hz}$.

The analysis compared data for identifying the most responsive stimulus from all three stimuli designs. Each set of data had 150 amplitude FFT values for each frequency for one participant. Each participant had six sets which included $70 \mathrm{~mm}, 90 \mathrm{~mm}, 110 \mathrm{~mm}, 130 \mathrm{~mm}$, concentric circles and horizontal stimuli. Figures 5 to 9 shows the box plots for the obtained maximal FFT amplitudes for $7 \mathrm{~Hz}$ stimulus from five participants for different radial sizes, concentric circles and horizontal stimuli. The central line shows the median value while the edges of the box are the 25th and 75th percentiles with the whiskers extending to the most extreme values.

The Kruskal-Wallis tests, $\chi^{2}(d f=5, N=150)$ showed significant differences between stimuli for all five participants:

$$
\begin{aligned}
& P 1: H=347.28, p=6.73 e^{-73} \\
& P 2: H=286.46, p=8.11 e^{-60} \\
& P 3: H=402.16, p=1.01 e^{-84} \\
& P 4: H=227.94, p=2.95 e^{-47} \\
& P 5: H=226.22, p=6.90 e^{-47}
\end{aligned}
$$

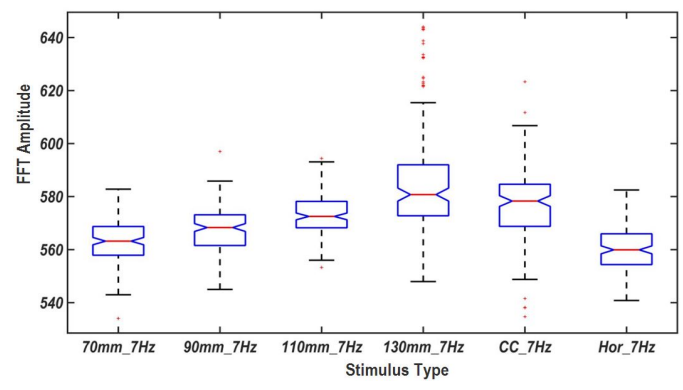

Fig. 5. Maximal FFT amplitude values from first participant 


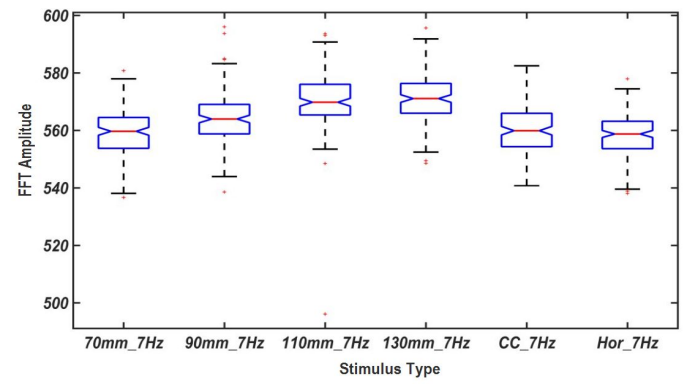

Fig. 6. Maximal FFT amplitude values from second participant

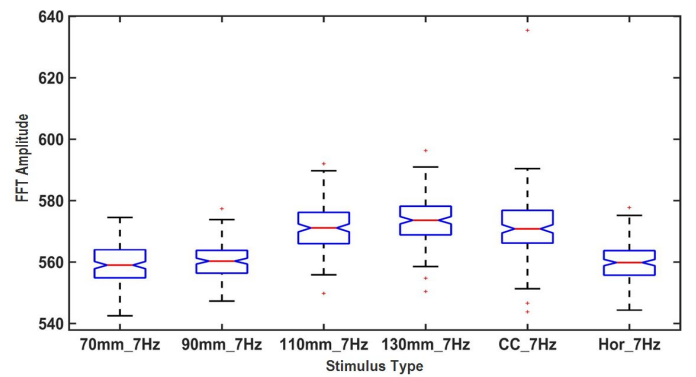

Fig. 7. Maximal FFT amplitude values from third participant

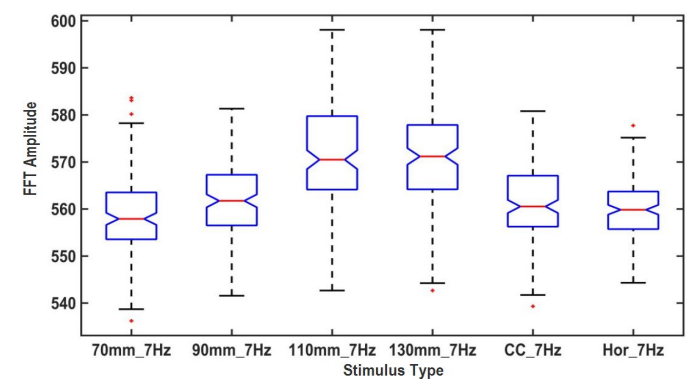

Fig. 8. Maximal FFT amplitude values from fourth participant

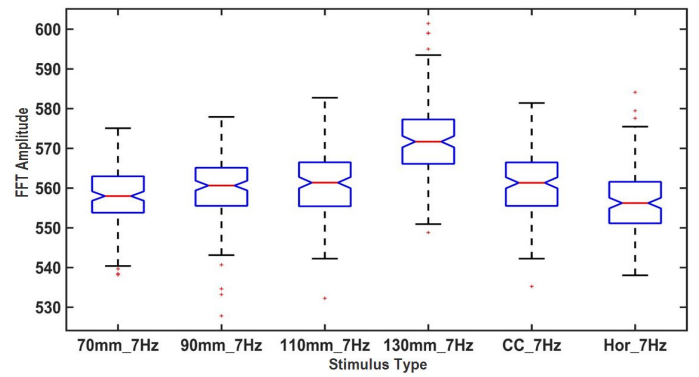

Fig. 9. Maximal FFT amplitude values from fifth participant
TABLE II

MEAN RANK FROM KRUSKAL-WALLIS TEST

\begin{tabular}{|c|c|c|c|c|c|}
\hline \multirow{2}{*}{$\begin{array}{c}\text { Stimulus } \\
\text { at } 7 \mathrm{~Hz}\end{array}$} & \multicolumn{5}{|c|}{ Participants (mean rank) } \\
\cline { 2 - 6 } & $\mathrm{S} 1$ & $\mathrm{~S} 2$ & $\mathrm{~S} 3$ & $\mathrm{~S} 4$ & $\mathrm{~S} 5$ \\
\hline $70 \mathrm{~mm}$ & 291.64 & 319.98 & 263.17 & 309.44 & 351.91 \\
\hline $90 \mathrm{~mm}$ & 397.38 & 455.04 & 288.88 & 404.93 & 417.44 \\
\hline $110 \mathrm{~mm}$ & 532.01 & 640.91 & 602.48 & 626.69 & 450.88 \\
\hline $130 \mathrm{~mm}$ & 668.68 & 650.52 & 665.96 & 634.02 & 721.62 \\
\hline Conc & 594.11 & 337.80 & 599.78 & 386.43 & 443.05 \\
\hline Hori & 219.16 & 298.74 & 282.71 & 341.48 & 318.08 \\
\hline
\end{tabular}

The mean ranks for all different visual stimuli for five subjects is shown in Table II. From these tables, it can be observed that $130 \mathrm{~mm}$ radial stimulus gave better performance compared with concentric and horizontal stimulus. The least performance was from the horizontal or $70 \mathrm{~mm}$ radial stimuli for all participants.

For further analysis of the SSVEP reponses, different sizes of radial stimuli, concentric circles and horizontal stimulus, data from all five participant were combined for each stimulus type. For each stimulus, 750 FFT amplitude values were used from five participants in Kruskal-Wallis test $(150$ values from each participant). Figure 10 shows the box plot representation of all stimuli from five participants. The highest SSVEP response was for $130 \mathrm{~mm}$ radial stimulus followed by $110 \mathrm{~mm}, 90 \mathrm{~mm}, 70 \mathrm{~mm}$, concentric circles and horizontal stimulus, confirming the values obtained in Table II and boxplots in Figures 5-9.

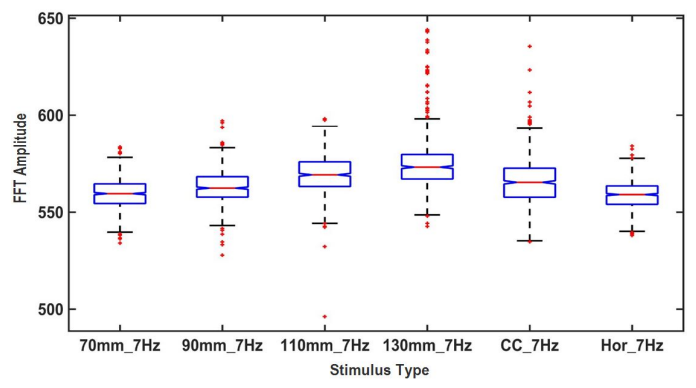

Fig. 10. Analysis of different sized radial, concentric circles and horizontal stimuli performances from all five participants

Analyses for other frequencies $(8,9$, and $10 \mathrm{~Hz})$ were performed using the same procedure for all the participant data. For each stimulus type, 750 FFT amplitudes values were used from all five participants for performing the Kruskal-Wallis test. Figures 11 to 13 show the box plots for frequencies 8,9 and $10 \mathrm{~Hz}$. The mean ranks for all different visual stimuli for five subjects and frequencies 8,9 , and 10 $\mathrm{Hz}$ are shown from Table III to Table V. The results showed the $130 \mathrm{~mm}$ radial stimulus exhibited better performance than concentric and horizontal visual stimuli, and horizontal being the least responsive stimulus.

The Kruskal-Wallis tests, $\chi^{2}(d f=5, N=750)$ showed significant differences between stimuli for all five participants 
for 8,9 and $10 \mathrm{~Hz}$ for the three different visual stimuli;

$$
\begin{gathered}
8 H z: H=911.021, p=6.81 e^{-196} \\
9 H z: H=1.48 e^{+03}, p=9.29 e^{-320} \\
10 H z: H=696.99, p=1.56 e^{-149}
\end{gathered}
$$

The lowest response was from the horizontal stimulus when compared with radial and concentric circles. In the radial ring comparison $130 \mathrm{~mm}$ had the highest performance and $70 \mathrm{~mm}$ responses were the lowest in all frequency ranges.

In the frequency analysis, $7 \mathrm{~Hz}$ showed the highest response as compared to 8,9 and $10 \mathrm{~Hz}$. Figure 14 shows the analysis of 750 FFT amplitude values for $130 \mathrm{~mm}$ stimulus for four frequencies. Table VI shows the mean rank for the analysis.

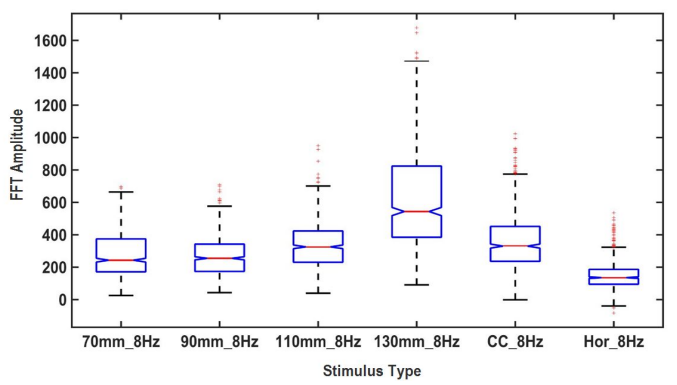

Fig. 11. Maximal FFT amplitude values from five participants for $8 \mathrm{~Hz}$

TABLE III

MEAN RANK FROM KRUSKAL-WALLIS TEST FOR ALL FIVE PARTICIPANTS AT $8 \mathrm{~Hz}$

\begin{tabular}{|c|c|c|c|c|c|}
\hline \multicolumn{6}{|c|}{ Participants (mean rank) } \\
\hline $70 \mathrm{~mm}$ & $90 \mathrm{~mm}$ & $110 \mathrm{~mm}$ & $130 \mathrm{~mm}$ & Conc & Hori \\
\hline 136.07 & 140.91 & 184.46 & 284.42 & 191.89 & 115.61 \\
\hline
\end{tabular}

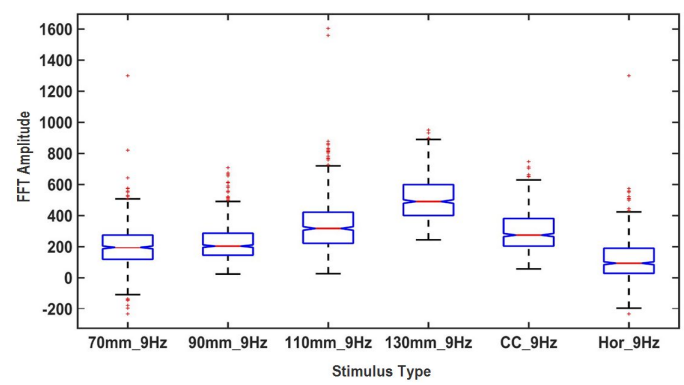

Fig. 12. Maximal FFT amplitude values from five participants for $9 \mathrm{~Hz}$

TABLE IV

MEAN RANK FROM KRUSKAL-WALLIS TEST FOR ALL FIVE PARTICIPANTS AT $9 \mathrm{~Hz}$

\begin{tabular}{|c|c|c|c|c|c|}
\hline \multicolumn{7}{|c|}{ Participants (mean rank) } \\
\hline $70 \mathrm{~mm}$ & $90 \mathrm{~mm}$ & $110 \mathrm{~mm}$ & $130 \mathrm{~mm}$ & Conc & Hori \\
\hline 114.32 & 128.51 & 204.37 & 305.92 & 184.55 & 102.36 \\
\hline
\end{tabular}

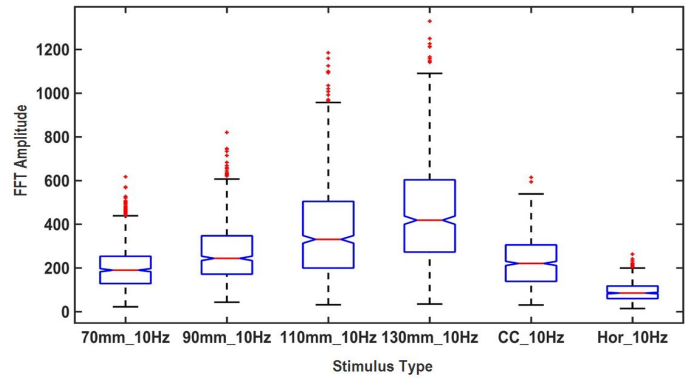

Fig. 13. Maximal FFT amplitude values from five participants for $10 \mathrm{~Hz}$

TABLE V

MEAN RANK FROM KRUSKAL-WALLIS TEST FOR ALL FIVE PARTICIPANTS AT $10 \mathrm{~Hz}$

\begin{tabular}{|c|c|c|c|c|c|}
\hline \multicolumn{5}{|c|}{ Participants (mean rank) } \\
\hline $70 \mathrm{~mm}$ & $90 \mathrm{~mm}$ & $110 \mathrm{~mm}$ & $130 \mathrm{~mm}$ & Conc & Hori \\
\hline 128.75 & 177.37 & 221.13 & 258.14 & 152.48 & 102.21 \\
\hline
\end{tabular}

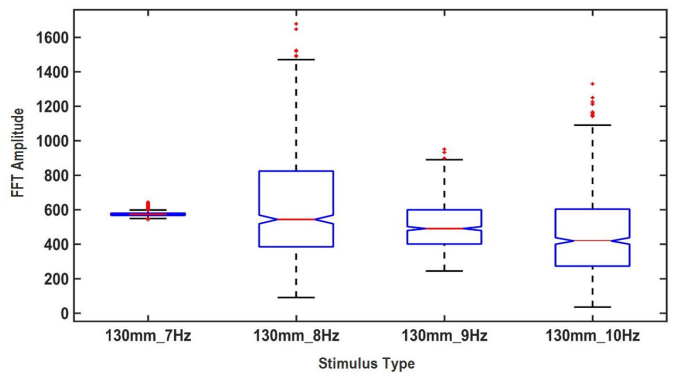

Fig. 14. Analysis of frequency response from all five participants for 130 mm radial stimulus

The Kruskal-Wallis tests, $\chi^{2}(d f=3, N=750)$ showed significant differences between frequencies $7,8,9$ and $10 \mathrm{~Hz}$ for all five participants $H=256.59, p=2.45 e^{-55}$.

Frequency $7 \mathrm{~Hz}$ had the highest response followed by 8,9 and $10 \mathrm{~Hz}$ and this confirms our previous findings [14].

TABLE VI

MEAN RANK OF FREQUENCY RESPONSE FROM KRUSKAL WALLIS TEST FOR ALL FIVE PARTICIPANTS FOR $130 \mathrm{MM}$ RADIAL STIMULUS

\begin{tabular}{|c|c|c|c|}
\hline \multicolumn{4}{|c|}{ Participants (mean rank) } \\
\hline $7 \mathrm{~Hz}$ & $8 \mathrm{~Hz}$ & $9 \mathrm{~Hz}$ & $10 \mathrm{~Hz}$ \\
\hline 182.89 & 163.34 & 138.11 & 115.58 \\
\hline
\end{tabular}

In visual stimulus, comfortability analysis for the participants, a scale of one to ten (ten being the most comfortable) was used for comparing the stimulus comfort with reduced visual fatigue. The response is shown as a bar chart in Fig 15. Even though only five participants completed the questionnaire, all participants responded $130 \mathrm{~mm}$ radial stimulus as the most comfortable flicker as compared to other visual stimuli. 


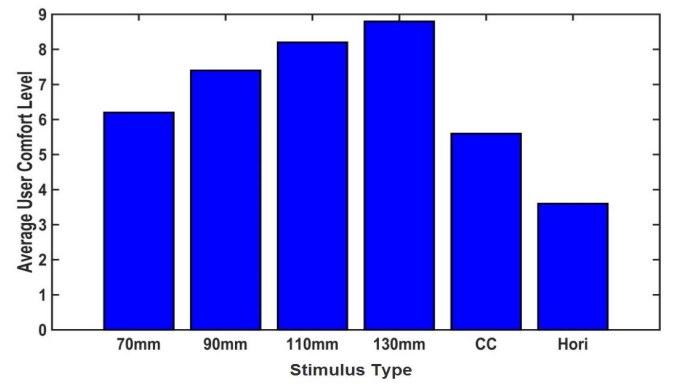

Fig. 15. Analysis of participant responses for stimulus comfortability

\section{CONCLUSION}

In this study, we investigated the influence of three different green COB LED visual stimuli to evoke SSVEP responses in brain. Comparisons were made between radial, concentric circles and horizontal stimuli that flashed at frequencies 7,8 , 9 , and $10 \mathrm{~Hz}$. From the analysis of data from five participants, radial stimulus exhibited the highest response for SSVEP in all participants followed by the concentric circles and horizontal stimuli. For the radial stimulus, the ring diameter analysis for all the participants revealed that $130 \mathrm{~mm}$ had the highest response followed by 110,90 and $70 \mathrm{~mm}$. The 70 $\mathrm{mm}$ radial gave better performance when compared with the horizontal stimulus.

Furthermore, the participants observed radial stimulus was better for viewing comfort as compared to concentric circles and horizontal stimuli. In addition, the $130 \mathrm{~mm}$ radial stimulus was more comfortable for viewing than $70 \mathrm{~mm}$ radial stimulus. Therefore, it is suggested that $130 \mathrm{~mm}$ radial stimulus is used to evoke SSVEP for BCI applications due to maximal response and better viewing comfort. Future research could explore the influence of various random and static patterns based on $\mathrm{COB}$ LEDs in evoking SSVEP responses in real time.

\section{REFERENCES}

[1] C. Cecere, C. Corrado, and R. Polikar, "Diagnostic utility of EEG based biomarkers for Alzheimer's disease," in Bioengineering Conference (NEBEC), 2014 40th Annual Northeast, 2014, pp. 1-2.

[2] N. N. Kulkarni and V. K. Bairagi, "Electroencephalogram based diagnosis of Alzheimer disease," in 9th International Conference on Intelligent Systems and Control (ISCO), IEEE, 2015, pp. 1-5.

[3] P. R. Pal, P. Khobragade, and R. Panda, "Expert system design for classification of brain waves and epileptic-seizure detection," in Student's Technology Symposium (TechSym), IEEE, 2011, pp. 187-192.

[4] L. Cao, J. Li, H. Ji, and C. Jiang, "A hybrid brain computer interface system based on the neurophysiological protocol and brain-actuated switch for wheelchair control," Journal of Neuroscience Methods, vol. 229, pp. $33-43,2014$.

[5] A. C. Lopes, G. Pires, and U. Nunes, "Assisted navigation for a brainactuated intelligent wheelchair," Robotics and Autonomous Systems, vol. 61, no. 3, pp. 245-258, Mar. 2013.
[6] L. Bi, J. Lian, K. Jie, R. Lai, and Y. Liu, "A speed and directionbased cursor control system with P300 and SSVEP," Biomedical Signal Processing and Control, vol. 14, pp. 126-133, 2014.

[7] P. F. Diez, S. M. T. Müller, V. A. Mut, E. Laciar, E. Avila, T. F. BastosFilho, and M. Sarcinelli-Filho, "Commanding a robotic wheelchair with a high-frequency steady-state visual evoked potential based braincomputer interface," Medical Engineering \& Physics, vol. 35, no. 8, pp. 1155-1164, 2013.

[8] L. F. Nicolas-Alonso and J. Gomez-Gil, "Brain computer interfaces, a review," Sensors, vol. 12, no. 2, pp. 1211-1279, 2012.

[9] S. Mouli, R. Palaniappan, I. P. Sillitoe, and J. Q. Gan, "Quantification of SSVEP responses using multi-chromatic LED stimuli: Analysis on colour, orientation and frequency," in Computer Science and Electronic Engineering Conference (CEEC), 2015 7th, Sept 2015, pp. 93-98.

[10] F.-B. Vialatte, M. Maurice, J. Dauwels, and A. Cichocki, "Steady-state visually evoked potentials: Focus on essential paradigms and future perspectives," Progress in Neurobiology, vol. 90, no. 4, pp. 418-438, 2010.

[11] M. Wang, I. Daly, B. Z. Allison, J. Jin, Y. Zhang, L. Chen, and X. Wang, "A new hybrid BCI paradigm based onP300 and SSVEP," Journal of Neuroscience Methods, vol. 244, pp. 16-25, 2015.

[12] Y. Zhang, G. Zhou, J. Jin, X. Wang, and A. Cichocki, "SSVEP recognition using common feature analysis in brain-computer interface," Journal of Neuroscience Methods, vol. 244, pp. 8-15, 2015.

[13] A. Cotrina, A. Benevides, A. Ferreira, T. Bastos, J. Castillo, M. L. Menezes, and C. Pereira, "Towards an architecture of a hybrid BCI based on SSVEP-BCI and passive-BCI," in 36th Annual International Conference on Engineering in Medicine and Biology Society (EMBC), IEEE. IEEE, 2014, pp. 1342-1345.

[14] S. Mouli, R. Palaniappan, I. P. Sillitoe, and J. Q. Gan, "Performance analysis of multi-frequency SSVEP-BCI using clear and frosted colour LED stimuli," in 13th International Conference on Bioinformatics and Bioengineering (BIBE),. IEEE, 2013, pp. 1-4.

[15] Z. Wu, Y. Lai, Y. Xia, D. Wu, and D. Yao, "Stimulator selection in SSVEP-based BCI," Medical Engineering \& Physics, vol. 30, no. 8, pp. 1079-1088, 2008.

[16] M. H. Chang, H. J. Baek, S. M. Lee, and K. S. Park, "An amplitudemodulated visual stimulation for reducing eye fatigue in SSVEP-based brain-computer interfaces," Clinical Neurophysiology, vol. 125, no. 7, pp. 1380-1391, 2014

[17] H.-J. Hwang, D. H. Kim, C.-H. Han, and C.-H. Im, "A new dualfrequency stimulation method to increase the number of visual stimuli for multi-class SSVEP-based brain-computer interface (BCI)," Brain Research, vol. 1515, pp. 66-77, 2013.

[18] M. Hu and Y. Wu, "Full-color LED display research based on chip on board (COB) package," in 15th International Conference on Electronic Packaging Technology (ICEPT). IEEE, 2014, pp. 97-100.

[19] Y.-T. Wang, Y. Wang, C.-K. Cheng, and T.-P. Jung, "Measuring steadystate visual evoked potentials from non-hair-bearing areas," in Annual International Conference of the IEEE on Engineering in Medicine and Biology Society (EMBC), IEEE, 2012, pp. 1806-1809.

[20] A. Delorme and S. Makeig, "Eeglab: An open source toolbox for analysis of single-trial EEG dynamics including independent component analysis," Journal of Neuroscience Methods, vol. 134, no. 1, pp. 9-21, 2004.

[21] Emotiv, Wearables for your brain, 2016 (accessed 13 March , 2016). [Online]. Available: http://www.emotiv.com. 